\title{
Molecular Simulation and Theory for Nanosystems: Insights for Molecular Motors
}

\author{
Richard J. Sadus \\ Centre for Molecular Simulation, Swinburne University of Technology, \\ PO Box 218, Hawthorn, Victoria 3122, Australia, rsadus@swin.edu.au
}

\begin{abstract}
Knowledge of self-assembling molecules such as DNA and dendritic systems can provide beneficial insights for the development of tailor-made nano-scale devices. Another important category of nanosystems is molecular systems that provide a mechanism of molecular propulsion such a myosin, kinesin and adenosine triphosphate synthase. Work on these so-called 'molecular motors' has been led largely by experimental investigations. The application of rigorous theoretical techniques such as molecular simulation is a considerable computational challenge. However, the reward for accepting the challenge can be a greatly improved theoretical insight, which benefits the development of nanotechnologies. In this work, we discuss recent progress in improving the theoretical understanding of an important molecular motor, adenosine triphosphate synthase.
\end{abstract}

Keywords: molecular simulation, molecular motors, nanosystems, adenosine triphosphate synthase

\section{INTRODUCTION}

A 'molecular motor' is a molecular system that is capable of exerting forces and motion at molecular and cellular scales. Molecular motors are expected to play an increasingly important role in nanotechnology by providing a means of controllable mechanics and functions [1] [2]. The ultimate goal of building an artificial molecular motor is well beyond current scientific and technical capabilities. In contrast, there are many naturally occurring biomolecules that work as molecular motors [3] [4] [5], which could provide useful insights into the design and construction of purpose-built systems. Arguably the most important class of molecular motors is ATP-fueled enzymes such as myosin, kinesin, and adenosine triphosphate synthase (ATPase) .

One of the most important [3] [4] molecular motors is $\mathrm{F}_{1} \mathrm{~F}_{0}$-ATPase (Figure 1). The $\mathrm{F}_{1} \mathrm{~F}_{0}$-ATPase motor is an enzyme protein complex, which is primarily responsible for the process of energy conversion in bacteria, chloroplasts and mitochondria. The two main sections of $\mathrm{F}_{1} \mathrm{~F}_{0}$-ATPasecontain multiple domains or subunits. The alternating domains, labelled $\alpha$ and $\beta$, are constructed as a hexamer around the linking central $\gamma \delta \varepsilon$ subunits. In common with many other molecular motors, $\mathrm{F}_{1} \mathrm{~F}_{0}$-ATPase is powered by the hydrolysis and synthesis reactions of adenosine triphosphate (ATP). Reactions involving ATP trigger the $(\alpha \beta)_{3}$ subunits into a series of asymmetrical and sequential transitions through different conformational and binding states causing the central $\gamma \delta \varepsilon$ subunits to rotate [3] [6]. It is this rotary motion that makes $\mathrm{F}_{1} \mathrm{~F}_{0}$-ATPase the most appealing [7] system to investigate because rotation is an essential feature of all macroscopic human-made motors. In contrast other molecular motors such as myosin [8], kinesin and dynein [9] exhibit only translational motion, which cannot be readily adapted for a general-purpose motor

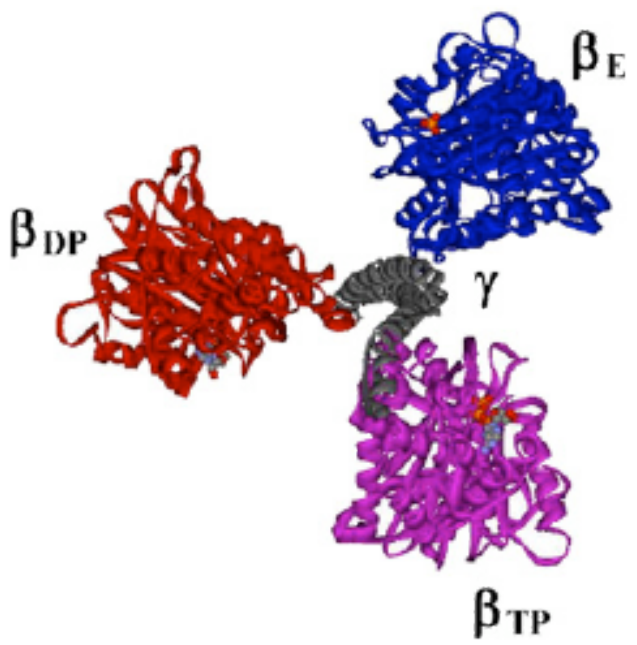

Figure 1: Schematic top view of the $\beta$ protein domains of the $\mathrm{F}_{1} \mathrm{~F}_{0}$-ATPase molecular motor.

Considerable efforts have been made to understand the behaviour of $\mathrm{F}_{1} \mathrm{~F}_{0}-\mathrm{ATPase}$. These include singlemolecular measurements [10] [11] [12] [13], and studies of both the functional microbiology [3] [14] and the structural biology [6] of the system. In addition, theoretical studies [15] have been reported. However, the exact molecular level details of the operation of ATPase remain largely unresolved [13]. The mechanism of ATP binding states, nucleotide reaction and release from the protein subunits 
represents an important challenge to our understanding of the functions of molecular motors. Our understanding is also deficient for critical molecular processes such as the binding of ATP to active subunits, reaction, catalytic pathways, the dynamic conformations of subunits and mechanical transduction processes.

In common with work on other nanosystems, our understanding of molecular motors has been led by experimental investigations. The application of rigorous theoretical techniques such as molecular simulation [16] is a computational challenge. However, the reward for accepting the challenge can be a greatly improved theoretical insight, which benefits the development of nanotechnologies. In this work, we review some of our recent progress in understanding ATPase.

\section{RESULTS AND DISCUSSION}

It is generally accepted that strong dynamically regulated conformational transitions and cooperativity are primarily responsible for the operation of the ATP-fuelled molecular motors. However, a molecular understanding of the mechanisms and underlying dynamics remains unclear [7] [17]. Efforts to determine the operating mechanism and dynamics of $\mathrm{F}_{1} \mathrm{~F}_{0}$-ATPase, include single molecular measurements [10] [12] [13], functional microbiology [3] [14], and structural biology [6] studies. Theoretical studies have also been reported [15] [18]. However, the exact molecular level mechanisms and underlying dynamics of $\mathrm{F}_{1}$-ATPase remain largely unresolved [14] [17]. As partially detailed below, our work [19] [20] [21] on molecular motors indicates that the critical deficiency is in our knowledge of molecular processes such as the binding of ATP to the active subunits, nucleotide reactions and catalytic pathways, the structural transitions of subunits and mechanochemical transduction processes. In particular, the main issues affecting molecular motors are dynamic fluctuations, correlations and allostery across different spatial and time scales.

In general, understanding the functioning of biomolecules requires knowledge of the relationships between structure, dynamics and functions, e.g. protein folding and enzymatic catalysis [22]. Recent studies suggest that dynamics plays critical roles in regulating certain protein functions via allostery and cooperativity [23] [24]. Therefore, the issues involved in understanding molecular motors are part of the broader challenges encountered in both protein dynamics and protein functions.

We [19] [20] [21] have made considerable progress in understanding the mechanism of molecular motors by studying $\mathrm{F}_{1}$-ATPase as a prototype molecular motor. $\mathrm{F}_{1^{-}}$ ATPase hydrolyses ATP into ADP and Pi and converts chemical energy into mechanical rotation. We have developed [19] [20] a computational model based on enzyme kinetics and Langevin dynamics that accounts for the chemomechanics of rotation and the load-dynamics of $\mathrm{F}_{1}$-ATPase. It was demonstrated that the torsional energy and stepwise rotation is regulated by a series of nearequilibrium reactions involving the binding and unbinding of nucleotides. The model also uses an effective "ratchet" drag coefficient and a chemomechanic coefficient. We have analysed the theoretical load-rotation profile when the motor drives an actin filament. As illustrated in Figure 2, comparison with experimental data indicates that this model is a reasonable approximation. It is capable of both qualitative and quantitative agreement with experiment.

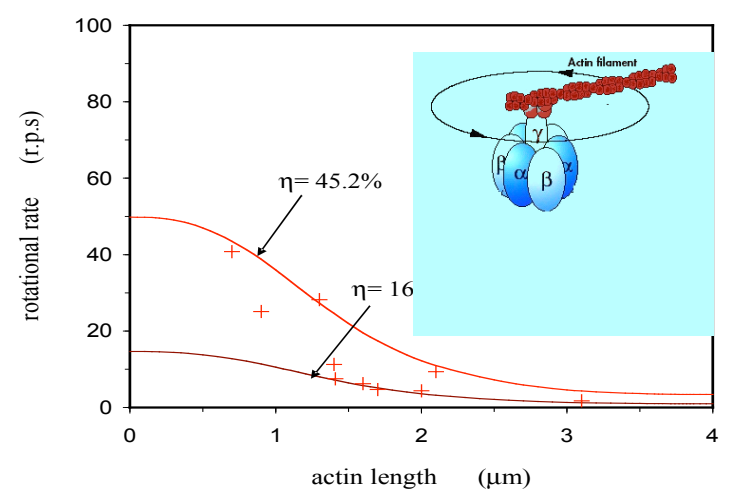

Figure 2: $\mathrm{F}_{1}$-ATPase drives an actin filament. The theoretical lines (red solid) indicate that the chemomechanical coefficients of the $\mathrm{F}_{1}$-ATPase motor fall between $0.165 \sim 0.452$ with an effective 'ratchet' coefficient of $1.07 \mathrm{pN} \mathrm{nm} \mathrm{s}$. The cross symbols are from single motor experiments. [19]

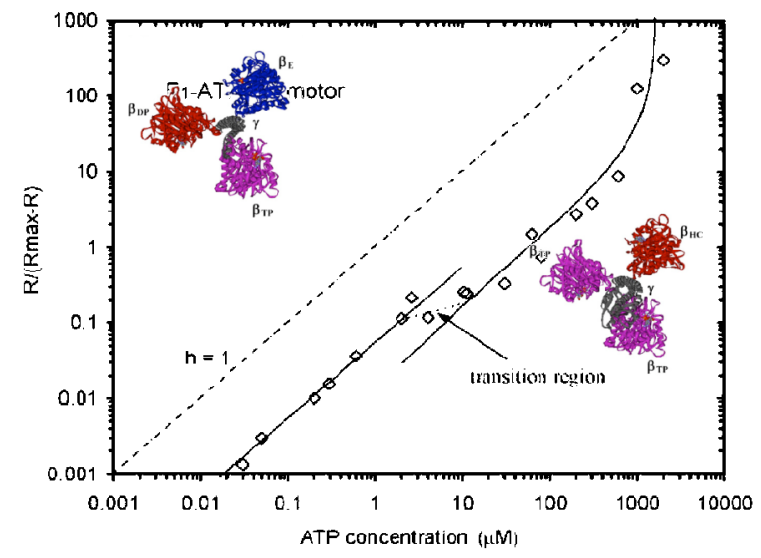

Figure 3: Cooperativity is a key dynamic feature for the steady operation of molecular motors. $\mathrm{F}_{1}$-ATPase with different cooperativity (diamonds are from motor experiments and solid lines are from our molecular simulation results) shows different rotation schemes of 'bisite' activities (Left) and 'tri-site' activities (Right). [21] 
The cooperativity of other ATP-fuelled molecular motors such as kinesin and myosin have also been analysed. Significantly, it was discovered [21] that the catalysis of these molecular motors does not follow a simple Michaelis-Menten mechanism (Figure 3). Instead motor activities such as the hydrolysis or processive rates of kinesin, myosin and $\mathrm{F}_{1}$-ATPase have a complex ATPdependent cooperativity. Our work indicates that conformational coupling of binding states of different subunits induces cooperativity.

In response to these insights, a conformation correlation theory of cooperativity has been developed [25] to predict this phenomenon in the ATP-fuelled motor proteins. In $\mathrm{F}_{1}$-ATPase, the rotation of the $\gamma \delta \varepsilon$ subunits against the hexameric $\alpha_{3} \beta_{3}$ subunits is highly coordinative, driven by ATP hydrolysis and structural changes at three $\beta$ subunits. However, the dynamical and coordinating structural transitions in the $\beta$ subunits are not fully understood at the molecular level. We examined structural transitions and domain motions in the active subunits of $\mathrm{F}_{1}$ ATPase via dynamical domain analysis of the $\alpha_{3} \beta_{3} \gamma \delta \varepsilon$ complex. The domain movement and hinge axes and bending residues were identified and determined for various conformational changes of the $\beta$-subunits. It was observed [25] that the P-loop and the ATP-binding pocket play essential mechanical functions, which is in addition to their catalytic roles. The cooperative conformational changes pertaining to the rotary mechanism of $F_{1}$-ATPase appears to be more complex than Boyer's 'bi-site' activity. These findings provide unique molecular insights into dynamic and cooperative domain motions in $\mathrm{F}_{1}$-ATPase.

\section{CONCLUSIONS}

The application of rigorous theoretical models and simulation provides valuable insights into the behaviour of nanosystems. Using such an approach, have identified complex cooperativity of ATP hydrolysis in $\mathrm{F}_{1}$-ATPase. Significantly, for the first time, activity cooperativity induced by conformational correlations was also found in other ATP-fuelled molecular motors, namely myosin and kinesin. We have also identified the dynamic and coordinating domain motions in the $\mathrm{F}_{1}$-ATPase molecular motor. These results illustrate the usefulness of theory in improving our fundamental knowledge of nanosystems.

\section{ACKNOWLEDGEMENTS}

This work was supported financially by the Australian Research Council as a Discovery Project.

\section{REFERENCES}

[1] National Nanotechnology Initiative (NNI) 2000: htttp://www.nano.gov

[2] M. Schliwa, and G. Woehlke, Nature 422, 759-765, 2006.

[3] P.D. Boyer, Annu. Rev. Biochem. 66, 717-749, 1997.

[4] W. Junge, Proc. Natl. Acad. Sci. USA 96, 47354737. 1999.

[5] H. Noji and M. Yoshida, Journal of Biological Chemistry 276, 1665-1668, 2001.

[6] R. I. Menz, J.E. Walker and A.G.W. Leslie, Cell 106, 331-341, 2001.

[7] M. Karplus, and Y. Q. Gao, Curr. Opinion Struct. Bio. 14, 250-259, 2004.

[8] J. A. Spudich, J. A., Nat. Rev. Mol. Cell Biol. 2, $387-$ 92, 2001.

[9] C. Kural, H. Kim, S. Syed, G. Goshima, V. I. Gelfand and P. R. Selvin, Science 308, 1469-1472, 2005.

[10] H. Noji, R. Yasusda, M. Yoshida, and K. Kinosita, K. Nature 386, 299, 1997.

[11] R. K. Soong, G. D. Bachand, H. P. Neves, A. G. Olkhovets, H. G. Craighead and C. D. Montemagno, Science 290, 1555, 2000.

[12] R. Yasuda, H. Noji, M. Yoshida, K. Kinosita, Jr and H. Itoh, H., Nature 410, 898, 2001.

[13] K. Shimabukuro, R. Yasuda, E. Muneyuki, K. Y. Hara, K. Kinosita and M. Yoshida, Proc. Natl. Acad. Sci. USA 100, 14731-14736, 2003.

[14] A. E. Senior, S. Nadanaciva and J. Weber, Biochim. Biophys. Acta 1553, 188-211, 2002.

[15] R. A. Bockmann, and H. Grubmuller, Nature Struct. Biol. 9, 198-202, 2002.

[16] R. J. Sadus, Molecular Simulation of Fluids: Theory, Algorithms and Object-Orientation, Elsevier, Amsterdam, 2002.

[17] Y. Q. Gao, W. Yang and M. Karplus , Cell 123, 195205, 2005.

[18] J. Ma and M. Karplus, Proc. Natl Acad. Sci. USA 95, 8502-8507, 1998.

[19] M. S. Liu, B. D. Todd and R. J. Sadus, J. Chem. Phys. 118, 9890-9898, 2003.

[20] M. S. Liu, B. D. Todd and R. J. Sadus, Biochim. Biophys. Acta 1698, 197-202, 2004.

[21] M. S. Liu, B. D. Todd and R. J. Sadus, Biochim. Biophys. Acta 1752, 111-123, 2005.

[22] M. Karplus, and J. Kuriyan, Proc. Natl. Acad. Sci. USA 102, 6679-6685, 2005.

[23] D. Kern and E. R. Zuiderweg, Curr. Opinion Struct. Bio. 13, 748-757, 2003.

[24] N. S. Popovych, Sun, R. H. Ebright and C. G. Kalodimos, Nature Struct. \& Mol. Bio. 13, 831 - 838., 2006. 
[25] M. S. Liu, B. D. Todd and R. J. Sadus, Biochim. Biophys. Acta 1764, 1553-1560, 2006. 